\title{
Qualitative Assessment of Contemporary Media Sharing Practices and Their Relationship to the sMS Platform
}

\author{
Maarten Wijnants \\ Expertise Centre for Digital Media \\ Wetenschapspark 2, 3590 Diepenbeek, Belgium \\ firstname.lastname@uhasselt.be
}

\author{
Jonas De Meulenaere Wendy Van den Broeck \\ IBBT-SMIT - Vrije Universiteit Brussel \\ Pleinlaan 9, 1050 Brussels, Belgium \\ jdemeule,wvdbroec@vub.ac.be
}

\begin{abstract}
Being a social species, humans sometimes desire to share information and to consume content as part of a group. A myriad of practical issues might however prevent people from physically congregating. The synchronous MediaSharing (sMS) framework attempts to remedy this inconvenience by supporting the real-time synchronization of multimedia content across dispersed locations. As such, it can be regarded as a social media consumption platform for geographically dislocated users. This paper presents the outcome of a two-fold prefatory qualitative assessment of the sMS application. A literature study was first conducted to gain insight in the contemporary (non-professional) practices of end-users with respect to the authoring, sharing and consumption of multimedia, as well as concerning their usage of digital social services like social networking sites. On the basis of focus group interviews, the findings from the desk research were then representatively verified and their relationship to the sMS framework was studied. The results reveal that users do recognize the need for an sMS-like application, that they generally appreciate its functionality and present feature collection, that the sMS service is largely compatible with or supplementary to their current habits, and that they would consider utilizing it in certain use cases.
\end{abstract}

\section{Categories and Subject Descriptors}

C.2.4 [Computer-Communication Networks]: Distributed Systems; H.5.1 [Information Interfaces And Presentation]: Multimedia Information Systems

\section{Keywords}

synchronous MediaSharing, sMS, qualitative evaluation, focus group research, social, media consumption

\section{INTRODUCTION}

One important form of social interaction is the communal consumption of multimedia [8]. Traditionally, social media

Permission to make digital or hard copies of all or part of this work for personal or classroom use is granted without fee provided that copies are not made or distributed for profit or commercial advantage and that copies bear this notice and the full citation on the first page. To copy otherwise, to republish, to post on servers or to redistribute to lists, requires prior specific permission and/or a fee.

SAM'12, October 29, 2012, Nara, Japan.

Copyright 2012 ACM 978-1-4503-1586-9/12/10 ...\$15.00. consumption entailed physical co-location of the involved persons. To some extent, these behaviors still exist today. For instance, it is still relatively common practice to recollect and relive shared experiences by jointly browsing through printed photographs. As another example, people continue to exhibit the tendency to assemble at a single location to watch the TV broadcast of an important sports event in the presence of each other. In recent years however, steady increases in Internet bandwidth and the continuously expanding multimedia features of (handheld) devices have rendered social multimedia sharing and consumption between non-colocated individuals or subgroups a reality.

It is in this Internet-based context that we have previously developed and proposed the synchronous MediaSharing (sMS) framework [19]. The fundamental objective of the sMS system consists of enabling geographically scattered users to share pictures and video clips with each other and to communally consume such multimedia content in a synchronous fashion. In an effort to emulate physical and social co-location, underlying synchronization mechanisms guarantee that all participants will view the same content at (approximately) the same time. Additional supportive tools like real-time speech communication and interfaces with social networking sites are provided to further improve the feeling of social presence among sMS users.

To direct the future technical development of the sMS platform, it is currently being subjected to a qualitative assessment. As part of this assessment, we have first performed a (non-exhaustive) literature study concerning (i) the nature of users' media capture and sharing behaviors, and (ii) the usage of online social networking services (SNSs), both in general and for media sharing purposes specifically. The outcome of this desk research will be presented in Section 2. Next, in Section 3 a concise overview of the feature set and the technical implementation of the sMS system will be given. Section 4 will then report on the results from focus group sessions in which we gauged participants' current media sharing practices and investigated how these fit in with the sMS service. Finally, our conclusions and future research directions will be communicated in Section 5 .

\section{LITERATURE REVIEW}

\subsection{Authoring and Sharing Practices}

\subsubsection{Photography}

Chalfen introduced the Kodak Culture concept to refer to the capture of particular domestic moments that are deemed 
photo-worthy, such as birthdays, parties or weddings [2]. The resulting photographs are shared only within intimate circles. In particular, the people featuring in the pictures are typically acquainted with the persons the pictures are shared with, and vice versa. Furthermore, such photo collections are generally accompanied with an oral narration, indicating that the pictures require contextual elucidation before they can be fully understood and appreciated [10]. Sarvas and Frohlich argue that domestic Kodak Culture photography holds the capacity to trigger memories and emotions and to create feelings of togetherness and belonging [15]. As such, they conclude that its value is quasi restricted to the intimate real-world social network it is a part of.

Largely contrasting the Kodak Culture model is the socalled snapr practice. The term was put forward by Miller and Edwards, who studied Internet-based picture sharing patterns by analyzing common behaviors on the popular photo sharing website Flickr [10]. Contrary to Kodak Culture practitioners, snaprs take pictures with the deliberate intention to share them and to showcase them not only to acquaintances, but just as importantly also to people outside their social graph. Snapr photos are typically also more artistic in nature, with the author in addition actively seeking acknowledgment for his creations. In an independently performed study, Murray uncovered the same type of behavior, yet denoted it with the term "serious amateurs" [12]. Miller and Edwards discovered that snapr practitioners tend to fully exploit the affordances Flickr provides like tagging and group formation, whereas Kodak Culture photographers exhibit stricter privacy concerns and are more likely to avoid the usage of the aforementioned functions [10].

The digitization of photography and the integration of digital cameras in consumer devices like smartphones has led to the emergence of a third type of picture authoring and sharing behavior: instant photography or so-called digital snapshots. Events one would not spend a picture on in the analog era are now being photographed without hesitation. People no longer only take pictures of events, but also of what Murray calls "one's discovery of the small and mundane" [12]. Kindberg et al. developed a taxonomy of six motivations to author images with a smartphone [7], including individual personal reflection and as a means to conduct tasks more efficiently. Some of these types of instant photographs are shot with the specific aim of sharing (e.g., snapshots that are intended to facilitate a mutual task), whereas others are originally not meant to be disseminated but might eventually still end up being circulated.

Research particularly pertaining to the sharing of photographs has also been described in the literature. Stelmaszewska et al. have established that people exhibit variable sharing behavior depending on the target audience, the location from which the picture is shared, and the value or significance a picture represents for its owner [16]. Moreover, they identified three triggers for the co-located sharing of pictures using cameraphones: motivational, contextual and ad hoc sharing. Pictures belonging to the motivational sharing category are shot with the deliberate purpose of sharing, meaning that the sharing is a preplanned activity. In the second category, the sharing of pictures emerges from practical context. For instance, while talking to someone, a picture could be exchanged and discussed. Finally, ad hoc sharing refers to the showing of a picture (e.g., a funny image) to someone without immediate cause or predefined motivation.

\subsubsection{Video}

The best-known and most universally used video sharing application is YouTube, which hosts a mix of user-generated and professional content. Ding et al. have examined the sharing activities of a sample of 300 YouTube users [3]. They distinguish between uploaders of self-generated content and of content (or fragments of it) that is copied from external sources like movies, TV shows or music videos. It was found that user-generated content sharers upload almost no copied content and vice versa. Furthermore, YouTube appears to be foremost exploited to disseminate copied content: $63 \%$ of the top uploaders chiefly share copied content. Quantitatively speaking, copied content uploaders also share more videos than user-generated content uploaders.

The sharing (and shared consumption) of digital video content has also been investigated in the context of social TV research. Here, the focus is exclusively on generating a shared experience by emulating the sense of simultaneously watching broadcast content for consumers that are temporally and/or spatially separated. Numerous social TV installations have been proposed and reported on in the last decade, yet none of them have proved to prevail or outgrow the research phase, despite some of them exhibited promising (tentative) results. Notable examples include Social TV 2 [5], CollaboraTV [13] and ConnecTV [17].

\subsection{Social Media}

Given its characteristics and top-level objective, the sMS service can be situated in the larger domain of social media [6]. SNSs, general-purpose ones like Facebook as well as those particularly devoted to the storage and sharing of multimedia such as Flickr, also belong to this application category. Rooted in the research areas of CSCW and groupware, social software hands users tools to interact, communicate and collaborate, but also allows them to pursuit the feeling of belonging to a group, for instance by facilitating the formation of virtual relationships. According to Sarvas and Frohlich, online SNSs provide powerful means to the area of domestic photography just because they let users get in touch with acquaintances easily, which in turn has proven to strengthen and reify social bonds [15].

Table 1 displays an adaptation of the traditional groupware taxonomy to so-called photoware, collaborative software that is centered around digital photography [4]. Typical online social software like SNSs are functionally restricted to the asynchronous sharing and consumption of media content, which places them in the "sending" quadrant of the typology. In contrast, the sMS framework can be situated in the "remote sharing" class, which connects it with telephony and instant messaging rather than existing photo (and video) sharing applications.

\subsection{Social Presence}

Social presence technology has been defined by Biocca et al. as digital mechanisms that are primarily intended to increase real-time social interaction [1]. It is apparent that the sMS application satisfies this predicate. Social presence involves three interrelated dimensions: co-presence, psychological involvement and behavioral engagement [1]. With respect to the sMS framework, co-presence might be obtained through the usage of voice chat and, in the case of the 3D NVE interface, by means of (customizable) avatars (see Section 3 for a discussion of these sMS assets). In ad- 
Table 1: Photoware typology; analog/digital technologies are printed in normal/italic font [4]

\begin{tabular}{lll}
\hline & SAME TIME & OTHER TIME \\
\cline { 2 - 3 } SAME & Prints, Slides \& projector & Shoeboxes, Albums \& frames \\
PLACE & CO-PRESENT SHARING & ARCHIVING \\
& Photo viewing software \& devices & CD-ROM, PC filestore, Photo website \\
\cline { 2 - 3 } & Telephone & Mail \\
OTHER & REMOTE SHARING & SENDING \\
PLACE & Application sharing, Instant mes- & Email attachment or website reference, \\
& saging, Video conferencing & Internet photo frames \\
\hline
\end{tabular}

dition, the visualization of the presence status of distant users (available, away, occupied, ....) is anticipated to considerably contribute to the co-presence dimension [5]; an implementation of such functionality is therefore currently ongoing. Discussing the shared content causes a psychological relationship to be established and it is expected that the mere act of synchronously consuming shared content will lead to psychological involvement. Finally, the sharing of media content itself, the speech-based communication and the interactions performed inside the 3D NVE can all be considered forms of behavioral engagement.

\section{SYNCHRONOUS MEDIASHARING}

The sMS framework is intended to afford geographically separated persons with a computer-mediated equivalent of socially sharing and synchronously consuming media content, which in reality is only feasible for physically co-located consumers [19]. In particular, via underlying inter-destination synchronization features, the framework guarantees that distributed participants are presented identical content at identical times. The flow of the content presentation is hereby not dictated by the framework, but instead is controlled by the users themselves. As such, the participants are fully empowered to determine the way and pace with which content is in real time shared and socially consumed.

\subsection{Architectural Design and Implementation}

The sMS system is an integrated framework consisting of a back-end plus a client-side API (see Figure 1). In terms of architectural design, the framework adheres to a client/server network topology. In this design, the server fulfills the role of centralized repository for session synchronization data. The server continuously monitors the state of active sMS sessions and stores it in a relational database in the form of synchronization instructions, which it in turn makes available for retrieval by clients. Combined, the server and database constitute the back-end of the framework. The client code on the other hand is responsible for interfacing with the end-user, for rendering the shared content, for initiating and controlling media sharing sessions, and for locally enforcing received synchronization instructions. To facilitate these processes, a client-side API is provided which enables users to interact with the sMS framework and the media sharing sessions they are presently engaged in.

Implementation-wise, the sMS system is characterized by its purely web-based approach. The framework exclusively relies on open, standardized web technologies and languages that are supported by the majority of contemporary operating systems, platforms and web browsers. The clientside API for instance is strictly developed in JavaScript. Some additional examples of exploited web standards include HTML 4 for user interface rendering, PHP for the

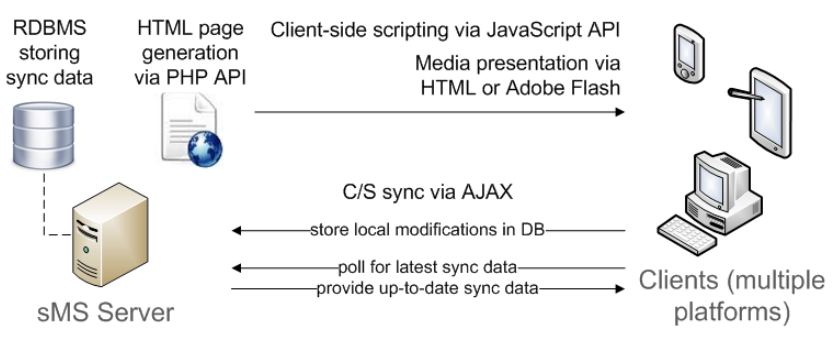

Figure 1: System architecture and technologies

server-side scripting and database interaction, AJAX for the client/server exchange of sMS session state and synchronization instructions, MediaRSS for the syndication of content, and HTML 4 plus Adobe Flash for the content presentation.

\subsection{Features}

The strict emphasis on web standards compliance represents an intentional design decision and entails three significant advantages. First, it maximizes the framework's market penetration and adoption potential by allowing it to cater to an amalgam of devices and platforms. Secondly, it automatically unlocks cross-platform media sharing options. Users owning divergent devices or hardware are perfectly capable of jointly engaging in a media sharing session. Lastly, it renders the service fit for integration in arbitrary digital environments and software, on condition that they support HTML browsing. As such, seamless content synchronization across the physical world and virtual spaces becomes feasible. This innovative feature is demonstrated in Figure 2, in which a cross-platform picture sharing session involving a tablet device and a desktop PC is illustrated. The tablet user participates through a standard web browser, whereas the desktop client presents the shared content within the confines of a native $\mathrm{C}++$ application, in particular a multiuser 3D Networked Virtual Environment (NVE).

With respect to content categories, the sMS system supports both digital images and video clips. In a picture sharing session, the inter-destination synchronization guarantees that, as users advance through the input set, the presently selected image is displayed at all participating sites. In the case of a video sharing setup, the synchronization is extended to the additional temporal aspect of this content type so that the playback time of the currently showing clip will also remain consistent for the involved parties.

Finally, the sMS framework incorporates a number of socially-inspired measures that are intended to enhance the sense of connectedness and presence for participants. Although these facilities do not contribute to the system's core functionality and in this sense can be regarded as being optional, their importance must not be underestimated as social aspects and stimuli might be indispensable factors for the end-user adoption of an online media sharing application [5]. A first such provided social feature is real-time interpersonal communication support in the form of voice-based conferencing. Secondly, the sMS system acknowledges the tremendous potential exhibited by online SNSs with regard to fostering social interaction between geographically dislocated people. The framework therefore has been interfaced with a number of such services that specifically focus on the dissemination of multimedia content, including Picasa Web Albums, Flickr, YouTube and Photobucket. Content that is available on these SNSs can be selectively filtered and subsequently exerted as input for sMS media sharing ses- 


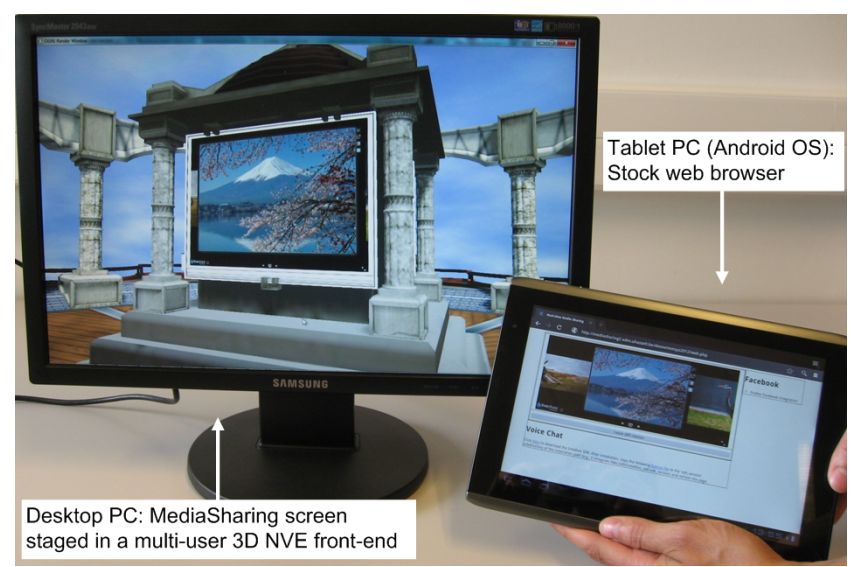

Figure 2: Cross-platform synchronization of picture content using heterogeneous front-ends

sions. Complementary to these multimedia-specific SNSs, the sMS framework supports interaction with the generalpurpose Facebook service. A client-side sMS API empowers users to post messages to their Facebook news feed, this way enabling them to communicate with acquaintances outside of the sMS system. An elective feature causes sMS-related actions and events (such as the local user joining or leaving a session) to trigger the automatic posting of Facebook messages with the intent to arouse end-user interest in the sMS service and to stimulate participation in ongoing sessions.

\section{FOCUS GROUP RESEARCH}

The literature review yielded interesting findings concerning the media sharing and consumption habits of (domestic) end-users. Focus group sessions were organized to further explore this topic and as an initial step in the qualitative evaluation of the sMS framework. In particular, the focus groups were intended to establish whether and how the sMS application fits in with prevailing media sharing practices, to address sMS-specific research questions, and to identify a series of functional and social requirements that will be taken into account in future revisions of the framework.

\subsection{Motivation}

A focus group is a qualitative research method in which test subjects are asked to discuss a particular topic in an interactive group setting. The motivations for adopting this specific form of user research for our study were numerous. First of all, focus group interviews are an efficient way to aggregate information from a group of people. Thanks to the direct interaction between moderator and respondents, the former can uncover deeper levels of meaning, make important links and identify subtle nuances in expression and meaning. Secondly, the methodology allows test subjects to express themselves in their own tone. Thirdly, the group context typically allows researchers to study people in a more natural, less formal setup than a one-to-one interview. An additional benefit of the interactive group setting is that it gives room for discussions among the participants; expressed opinions can be immediately reacted upon by others and may generate unanticipated issues and findings [9]. Finally, focus group interviews have been proven to be useful for introducing and discussing new services or technologies, since the group interaction yields data and insights that would be less accessible in an individual setting [11].

\subsection{Participant Demography}

Data was gathered from a total of 23 test subjects (12 male, 11 female) who were evenly divided over three separately organized focus group sessions. The average participant age per focus group was 21.9, 28.9 and 43.4, yielding an overall average age of 31.4. Purposeful sampling [14] on the basis of 3 criteria was applied when recruiting the participants: the extent to which they utilize social media platforms in general and Facebook in particular to share media content, and their degree of experience with $3 \mathrm{D}$ virtual environments. By deliberately targeting heterogeneous user profiles, we were able to gain insights in the current practices and expectations of a wide range of users; moreover, it contributed to the representativeness of the obtained results.

\subsection{Methodology}

Using the findings from the literature study as a staring point, a topic list for the focus groups was formulated. The moderator adhered to this topic list during the distinct sessions, which led to consistent, semi-structured interviews. It was clearly communicated to the participants that we were interested in finding out people's opinions and that there consequently were no right or wrong answers.

Each of the focus group interviews lasted 2 hours and conceptually comprised 2 distinct phases of equal length. In the first period, the users were asked to discuss their current practices related to content generation, social media usage and multimedia content sharing, as well as their experiences with (3D) virtual worlds. After approximately one hour, the focus was shifted to sMS-specific topics. This second phase started off with a short demonstration of the sMS framework in order to give the respondents an understanding of the application's rationale, mode of operation and features. The demonstration also served as an elicitation tool as well as to enrich and stimulate the following discussion, which was concentrated on the test subjects' opinions and expectations regarding the utilization of the sMS framework and how this would align with their present practices. In a concluding round, each participant was given the opportunity to state what he or she (dis)liked about the application.

\subsection{Qualitative Findings}

\subsubsection{Current Practices}

The photo- and video-production behaviors reported on by the focus group participants are largely in line with existing research on digital authoring practices. It appears that, besides classical opportunities such as weddings, family gatherings or holidays, spontaneous events while spending time with friends are also increasingly deemed captureworthy. A third authoring practice that emerged from the focus group interviews involves functional recordings (e.g., taking a photograph of a timetable). It was confirmed by the test subjects that memory evocation and the emotional capacity that is attached to pictures and videos exists in the first and second production category. An association with the Kodak Culture notion can be made here.

The content our participants indicated to share online, is a combination of personal, self-generated data (mainly pictures) and third party material. Provided examples of personal content included pictures that align with the Kodak Culture concept (e.g., family gatherings, daytrips with friends, holidays), but also photographs pertaining to for in- 
stance parties or hobbies. Third party content on the other hand refers to content made by people that do not have a direct relation with the person who shares it. In this regard, the test subjects indicated that they tend to share content from YouTube or news websites, but also for instance movie captures or funny comics. It was established that these various types of content have variable sharing qualities. For example, the interviews revealed that pictures or videos of parties or daytrips with friends are typically fit for asynchronous online dissemination through Facebook without further explanation, whereas recordings of family gatherings or holidays are often part of a larger narrative and would therefore benefit substantially from a synchronous sharing setup.

Three motivations for sharing personal content in an online environment were recorded in the focus group data. A first, involving Kodak Culture-like content, is social bonding. Distributing user-generated content allows people to share emotions and recall memories, this way strengthening social ties. A second motivation is reaction elicitation from the audience with whom the content is shared. This kind of identified behavior appears to align with the aforementioned snapr practice, as the focus group panel stated that in this context only aesthetically pleasing pictures are shared among Facebook friends. Finally, the respondents indicated that content was sometimes also shared for self-expression and self-presentation purposes [18]. Central to these phenomena is image building of the sharer towards his audience, which is considered to be especially relevant in an SNS environment such as Facebook. The content that is shared in this regard is not confined to user-generated items, but extends to third party data. Furthermore, the use of functionalities like untagging and the removal of "unfit" content from personal pages was shown to be correlated with the self-expression and -presentation motivation. In our data it is indicated that such curating behavior also influences the decision of how (personal) content will be shared.

The focus group results suggest that content is primarily shared within existing social networks, either in offline scenarios or by means of online applications. The online media sharing services that appear in our data set are highly divergent, ranging from e-mail and instant messaging over cyberlockers to general-purpose (e.g., Facebook) and dedicated (e.g., Picasa) SNSs. Respondents specified that their selection of an application for sharing content in an online environment depends on the nature of the content, the affordances of the application and the expected audience. For example, the test subjects acclaimed Facebook's ability to easily share personal content with all friends simultaneously and in an asynchronous fashion. This observation however only applied to certain content types (e.g., informal or noncommittal images), while in other contexts the use of Facebook was contested, chiefly because of the perceived lack of control it offers over one's personal content. For this reason, Kodak Culture-style photos were generally deemed inappropriate for being hosted on Facebook. In line with the Kodak Culture characteristics, the respondents indicated that they typically shared such photos via applications that afford regulation of the potential audience of the pictures. Because of its one-to-one communication model, e-mail was in this respect often mentioned, as was the use of password protected albums on online photo-hosting services like Picasa, the use of a shared dropbox folder or the creation of a private album or group on Facebook. In general, the utilized online applications that emerged from the focus group research are asynchronous in nature and appear to address different needs than those pursued by the sMS service.

\subsubsection{Assessing the sMS Service's Affordances}

With respect to the sMS framework, the focus group interviews zoomed in on its suitability regarding potential content types and also on the question how it might fit into users' current media sharing practices. The design of the application's user interface, either the 2D web interface or 3D NVE front-end, falls outside the scope of this research.

A number of interesting sMS use cases surfaced during the focus group sessions, the majority of which were situated in a residential context. In general, the sMS service was considered a means to share content and to strengthen existing offline social ties in a way that is largely supplementary to contemporary practices as well as existing media sharing applications. An important participant selection criterion is that respondents nearly exclusively envisaged themselves using the sMS application in combination with people that have a direct interest in the shared content. Furthermore, geographically dislocated family members or close friends were deemed most valuable as session participants. In contrast, the focus group data discloses that synchronously sharing content with casual acquaintances might be too tedious for users and could as such even lead to user frustration.

In terms of content compatibility, some restrictions emerged from the qualitative data. Not all content is expected to exhibit identical sharing qualities when used in combination with the sMS service. An apparently major requisite is that the content demands further discussion, either for the purpose of elaboration or modification (e.g., when discussing a collaborative drawing). For instance, pictures of occasional parties are less likely to be synchronously shared and discussed than a digital album of a wedding. Regarding privacy, the evaluation of the sMS framework was largely positive. A considerable fraction of respondents praised the fact that the session-based methodology empowers users to precisely decide with whom content is shared. In the same context, they voiced that the sMS service might serve as an answer to the aforementioned perceived lack of control over one's personal information, as is for instance the case with Facebook sharing (see Section 4.4.1).

The topic of initiating sMS sessions was also treated in the focus groups. By applying the framework from Stelmaszewska et al. (see Section 2.1.1) to the collected qualitative data, it was discovered that a sMS session will either be set up because of the content itself (motivational sharing) or will emerge from a particular situation (contextual sharing). The former recalls the notion of typical slide show presentations, the latter alludes to browsing trough a physical photo album as part of a larger conversation. The two identified session initiation motivations appear to involve different types of user behavior. In particular, the focus group research clarifies that sMS-mediated motivational sharing is appointment-driven, whereas in the contextual sharing use case the sMS platform can be regarded as an extension of online voice- or text-based chat practices. In the current implementation, the sMS service exclusively relies on its Facebook integration to engage potential users and to attract people to ongoing sessions. This functionality is more in line with contextual sharing than with motivational sharing. Incorporating adequate means to schedule sMS sessions is hence 
highly advisable and is a good example of a hiatus that was identified by the focus group research.

In sum, the focus group panel recognized the synchronous sharing of media content to be the fundamental quality and principal asset of the sMS service that clearly distinguishes it from currently used digital media sharing platforms. The flip side of the coin is that the synchronous nature of the service is also expected to raise restrictions in terms of the profile of appropriate sMS session participants and the types of content that are deemed share-worthy.

\section{CONCLUSIONS AND FUTURE WORK}

This paper has reported on our initial efforts at qualitatively gauging the sMS framework, a socially-aware media sharing platform that strives to overcome spatial disparity among consumers by offering inter-destination synchronization options for both picture and video content. The assessment took the form of focus group research, where the topic list for the group interviews was formulated on the basis of findings from an exploratory literature study. The focus group sessions yielded representative insights in contemporary non-professional trends and expectations regarding the sharing of content through dedicated digital applications in social media environments. At the same time, these trends were related to the sMS service and sMS-specific open research questions were discussed, such as the type of media content with which the service is deemed (most) compatible. In brief, the results reveal the lasting popularity of Kodak Culture-related practices in domestic contexts and establish that sMS use cases that center around this type of content are expected to be exceptionally fruitful. A sMS trait that was especially appreciated by the test subjects was the extensive level of control the application offers over the sharing of (potentially private) data. Finally, it has also been determined that the interviewed consumers consider the sMS tool to be largely complementary to their existing practices and currently employed content dissemination solutions, primarily due to sMS's synchronous character. All in all, the outcome of the focus group research leads us to cautiously conclude that the framework deserves its place in the digital media consumption and sharing landscape.

The presented user research only represents the initial phase of the qualitative evaluation of the sMS framework. As an example, some focus group participants have hinted at expectations of enhanced social presence, for instance when using the service with family members abroad. Unfortunately, the focus group methodology is unfit to decisively investigate evoked feelings of presence, as the methodology does not entail users gaining hands-on experience with the application by actually experimenting with it. The same is true for the witnessed sense of immersion. Therefore, another round of user research is scheduled in which we will let confined groups of users (e.g., family members) test the application with personal pictures in a controlled lab environment. Also, user experience research on the basis of the co-design methodology will be performed to elaborate and objectify the findings reported on in this article, as well as to expose missing features in the current sMS implementation.

\section{Acknowledgments}

Part of this research was performed in the context of IBBT project 3DTV 2.0. This project is co-funded by the IBBT
(Interdisciplinary institute for Broadband Technology), a research institute founded by the Flemish Government. Companies and organizations involved in the project include ANDROME and TP Vision, with project support of IWT.

\section{REFERENCES}

[1] F. Biocca, C. Harms, and J. K. Burgoon. Toward a More Robust Theory and Measure of Social Presence: Review and Suggested Criteria. Presence: Teleoperators and Virtual Environments, 12(5):456-480, Oct 2003.

[2] R. Chalfen. Snapshot Versions of Life. Bowling Green State University Popular Press, 1987.

[3] Y. Ding, Y. Du, Y. Hu, Z. Liu, L. Wang, K. Ross, and A. Ghose. Broadcast Yourself: Understanding YouTube Uploaders. In Proceedings of IMC 2011, pages 361-370, Berlin, Germany, Nov 2011.

[4] D. Frohlich, A. Kuchinsky, C. Pering, A. Don, and S. Ariss. Requirements for Photoware. In Proceedings of CSCW 2002, pages 166-175, New Orleans, LA, USA, Nov 2002.

[5] G. Harboe, C. J. Metcalf, F. Bentley, J. Tullio, N. Massey, and G. Romano. Ambient Social TV: Drawing People into a Shared Experience. In Proceedings of CHI 2008, pages 1-10, Florence, Italy, Apr 2008.

[6] A. M. Kaplan and M. Haenlein. Users of the World, Unite! The Challenges and Opportunities of Social Media. Business Horizons, 53(1):59-68, Jan/Feb 2010.

[7] T. Kindberg, M. Spasojevic, R. Fleck, and A. Sellen. I Saw This and Thought of You: Some Social Uses of Camera Phones. In Extended Abstracts CHI 2005, pages 1545-1548, Portland, OR, USA, Apr 2005.

[8] J. Lull. Inside Family Viewing: Ethnographic Research on Television's Audiences. Routledge, 1990.

[9] C. Marshall and G. B. Rossman. Designing Qualitative Research. Sage Publications, 2006.

[10] A. D. Miller and W. K. Edwards. Give and Take: A Study of Consumer Photo-Sharing Culture and Practice. In Proceedings of $\mathrm{CHI} 200 \%$, pages 347-356, San Jose, CA, USA, Apr 2007.

[11] D. L. Morgan. Focus Groups as Qualitative Research. Sage Publications, 1997.

[12] S. Murray. Digital Images, Photo-Sharing, and Our Shifting Notions of Everyday Aesthetics. Journal of Visual Culture, 7(2):147-163, Aug 2008.

[13] M. Nathan, C. Harrison, S. Yarosh, L. Terveen, L. Stead, and B. Amento. CollaboraTV: Making Television Viewing Social Again. In Proceedings of uxTV 2008, pages 85-94, Silicon Valley, CA, USA, Oct 2008.

[14] M. Sandelowski. Sample Size in Qualitative Research. Research in Nursing \& Health, 18(2):179-183, Apr 1995.

[15] R. Sarvas and D. M. Frohlich. From Snapshots to Social Media - The Changing Picture of Domestic Photography. Springer, 2011.

[16] H. Stelmaszewska, B. Fields, and A. Blandford. The Roles of Time, Place, Value and Relationships in Collocated Photo Sharing with Camera Phones. In Proceedings of BCS-HCI 2008, pages 141-150, Liverpool, UK, Sept 2008.

[17] H. Stokking, M. O. van Deventer, O. Niamut, F. Walraven, and R. Mekuria. IPTV Inter-Destination Synchronization: A Network-Based Approach. In Proceedings of ICIN 2010 pages 1-6, Berlin, Germany, Oct 2010.

[18] N. A. Van House, M. Davis, Y. Takhteyev, M. Ames, and M. Finn. The Social Uses of Personal Photography: Methods for Projecting Future Imaging Applications. In University of California Berkeley Working Papers, Sept 2004.

[19] M. Wijnants, J. Dierckx, P. Quax, and W. Lamotte. synchronous MediaSharing: Social and Communal Media Consumption for Geographically Dispersed Users. In Proceedings of MMSys 2012, pages 107-112, Chapel Hill, NC, USA, Feb 2012. 